\title{
9 Zur ersten Meta-Frage: Künftige Grabungen in der Villa und neue Papyri
}

Ein weiser Mann, der sich nicht brauchen lässt, und ein vergrabener Schatz - wozu sind die beiden nütze?

Jesus Sirach 20,32

\subsection{Zweite Wiederentdeckung der Villa 1986 und anschließende Ausgrabungen}

Schon im 19. Jahrhundert wurden Teile Herkulaneums unter freiem Himmel ausgegraben, aber erst unter der Leitung des Archäologen Amadeo Maiuri (1924-1961) wurde das Areal der Kleinstadt großflächig freigelegt. ${ }^{103}$ Die Ortschaft über den Ausgrabungen heißt seit 1969 „Ercolano“, was „Resina“ als Namen ablöste. Besonders sind Ausgrabungen im Gebiet des antiken Hafengeländes von Herkulaneum zu erwähnen, bei denen 1982 auch die berühmten 250 Skelette in den Bootshäusern gefunden wurden. Bis dahin hatte man angenommen, dass fast alle Bewohner Herkulaneums rechtzeitig fliehen konnten. ${ }^{104}$

Marcello Gigante formulierte leicht verklausuliert im ersten Band der Cronache Ercolanesi 1971 den Wunsch nach erneuten Grabungen in der Villa dei papiri. ${ }^{105}$ Nach Machbarkeitsstudien und Diskussionen zur Herangehensweise wurde die Villa, deren exakte Lokalisation beinahe in Vergessenheit geraten war, 1986 im Nordwesten Herkulaneums wiederentdeckt. Dabei bediente man sich alter Stollen und Schächte aus der Zeit der Bourbonischen Ausgrabungen (3.1). Erst zwischen 1994 und 1998 wurden Teile der Villa dei papiri unter freiem Himmel ergraben, vornehmlich das Atrium und angrenzende Räume. Diese erste Phase der Wiederentdeckung („Scavi Nuovi“) ist besonders mit Antonio de

103 Vgl. Guidobaldi, „Schatzgräber und Archäologen“ (wie Anm. 23), 21-22.

104 Luigi Capasso/Antonietta Di Fabrizio/Elisabetta Michetti/Ruggero D’ Anastasio, „Die Flüchtlinge am Strand. Die Untersuchungen der Skelette aus den Bootshäusern,“ in Mühlenbrock und Richter (Hgg.), Verschüttet vom Vesuv (wie Anm. 23), (45-55).

105 Marcello Gigante, „Premessa,“ CErc 1 (1971): (5). Eine interessante Randnotiz ist übrigens, dass Jean Paul Getty (s. 3.2) selbst erwog, sich finanziell bei erneuten Grabungen zu engagieren, aber der Bau der Getty-Villa bereits zu viele Gelder verschlungen hatte, vgl. Longo Auricchio et al., La villa (wie Anm. 5), 40. 
Simone verbunden. ${ }^{106}$ Ein zentrales Ergebnis dieser und folgender Grabungen unter Maria Paola Guidobaldi und Domenico Esposito (2007-2008) war, ${ }^{107}$ dass unter dem „Erdgeschoss“, d.h. der Ebene, welche die eigentliche Villa auf Webers Plan darstellt, noch mindestens zwei Etagen als Unterbau konstruiert waren, von denen die erste untere Etage im Bereich des Atriums offenbar als echte Wohnetage ausgestaltet war. Auch im Eingangsbereich der Villa fand man weitere Gebäudestrukturen, etwa ein „Schwimmbad“. Das Anwesen war teils in den Hang gebaut und die unteren, stützenden Etagen wohl dementsprechend angepasst. Die Villa dei papiri war somit noch weitaus ausladender (insbesondere hinsichtlich der Höhe) als bisher angenommen, was für die Frage nach möglicherweise unentdeckten Papyri verheißungsvoll ist. ${ }^{108}$ Sollte man wirklich bei Beginn der Eruption versucht haben, Bücher in Sicherheit zu bringen, wäre es eigentlich naheliegend gewesen, sie vor dem Ascheregen oder bodennahem Nebelrauch in die unteren Etagen zu tragen. Desungeachtet steht zu vermuten, dass in den unteren Etagen weitere Rollen - seien es dokumentarische Papyri, das „Archiv“ des (der) Villenbesitzer oder weitere literarische Papyri - deponiert waren. Bei den neuen Grabungen stieß man noch nicht in die Bereiche vor, wo vor etwa 250 Jahren die meisten Papyri gefunden wurden. Allerdings sind diese Bereiche teils durch Stollen zugänglich. Hinter der „Stanza V“, wo Philodems Bibliothek gefunden wurde, liegen noch einige Räume, die von Weber kaum erschlossen wurden. Viele dieser Räume sind heute nur wenige Meter von dem schon ausgegrabenen Areal entfernt.

106 Für eine Übersicht zur zweiten „Wiederentdeckung“ und den wichtigsten archäologischen Ergebnissen siehe Antonio de Simone, „Rediscovering the Villa of the Papyri,“ in Zarmakoupi (Hg.), The Villa (wie Anm. 5), (1-20).

107 Maria Guidobaldi und Domenico Esposito, „New Archaeological Research at the Villa of the Papyri in Herculaneum, “ in Zarmakoupi (Hg.), The Villa (wie Anm. 5), (21-62).

108 Siehe zu den Ergebnissen der jüngeren Grabungen etwa kompakt Domenico Camardo, „Recent Excavations in the Villa dei Papiri: 1990s-2008,“ in Lapatin (Hg.), Buried by Vesuvius (wie Anm. 5), (105-113). 


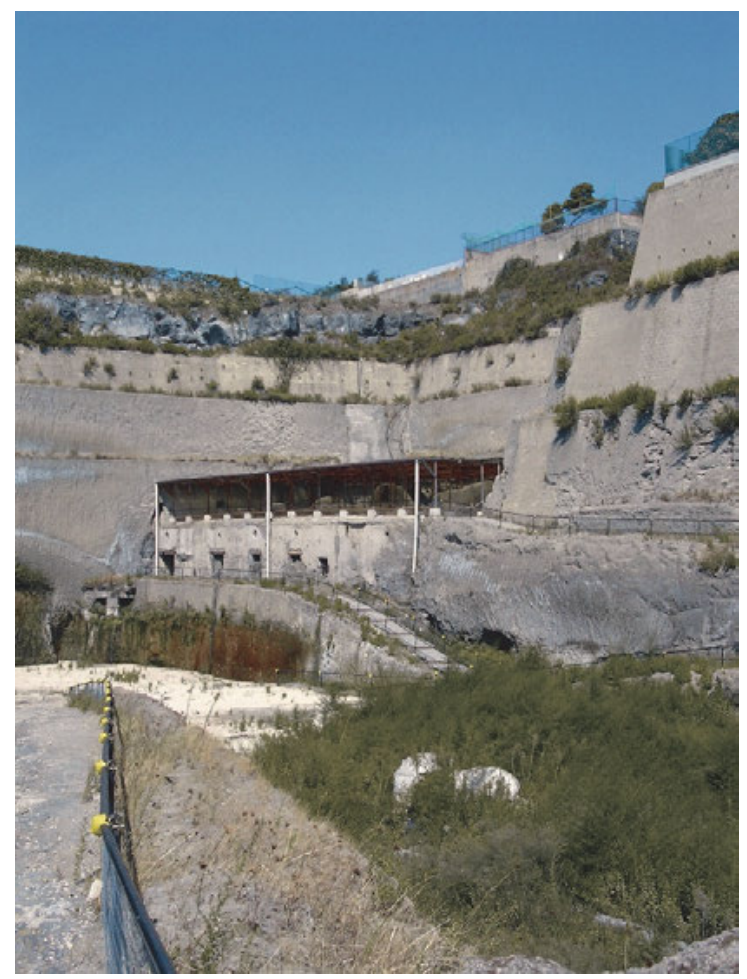

Abb. 26: Ausgrabungen

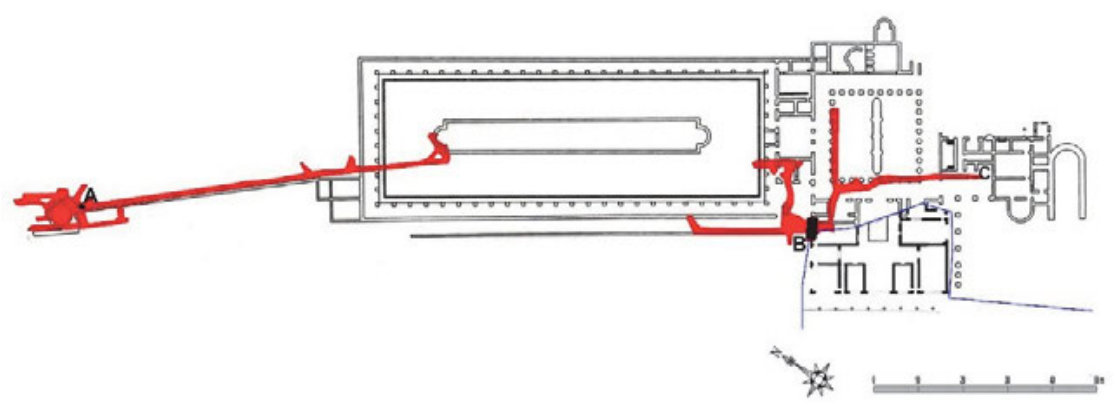

Abb. 27: Villenplan. Die blaue Linie markiert den Ausgrabungsbereich „sub caelo“, während die roten Linien alte Stollen anzeigen. In dem mit „C“ markierten Raum wurde Philodems Bibliothek gefunden. 


\subsection{Künftige Grabungen mit Suche nach Papyri?}

Es ist eine mitunter hitzig debattierte Frage zwischen Philologen/Papyrologen und Archäologen, ob weiter in der Villa mit der (Neben)Intention neuer Papyrusfunde gegraben werden sollte. Viele Archäologen sind skeptisch und verweisen auf die ohnehin schon zahlreichen ausgegrabenen, zu konservierenden Gebäude in Herkulaneum. Aus nachvollziehbaren methodischen Grundsätzen hegen sie eine natürliche Abneigung gegen jedwede Form von gezielter „Schatzsuche“. Standardmäßig graben Archäologen schichtenweise von oben nach unten, was aber im Falle der Villa wegen der besonderen Lage und der Verschüttungsverhältnisse kaum möglich ist. Jedoch steht diesem Aspekt mildernd entgegen, dass aufgrund der vulkanischen Überschüttungswellen in den oberen Schichten ohnehin nicht mit irgendwelchen (antiken) Gegenständen zu rechnen wäre. Auch liegen heute größere Teile der Villa nicht über bewohntem Gebiet von Ercolano, was für etwaige Grabungen von Vorteil wäre.

Die meisten Philologen können die Argumente der Archäologen nachvollziehen, erinnern aber an das große Potential für neue Papyrusfunde und deren weitreichende Bedeutung, derweil niemand negiert, dass die Frage noch unentdeckter Papyri auch mit vielen Unsicherheiten behaftet ist: Vom Fund keiner oder nur einer weiteren Rolle bis zum Fund tausender Papyri in einer weiteren griechisch, nicht-epikureischen oder lateinischen Bibliothek ist alles denkbar. Jedenfalls ist die Vorstellung verlockend, dass mit vergleichsweise wenig Aufwand die Bergung eines der vielleicht größten Schätze der antiken Welt durchführbar wäre. Es ist zu wünschen, dass die verantwortlichen Archäologen und Leiter einen innovativ-methodischen Weg finden, welcher - womöglich unter sinnvollem Einbezug schon vorhandener, alter Tunnel und natürlich unter Wahrung modern-archäologischer und konservatorischer Kriterien - zumindest eine genauere Erkundung relevanter Räume um das kleine Peristyl herum erlauben würde. Eine Harmonisierung von archäologisch-konservatorischen Ansätzen und bezüglich der Papyri bedingt zielgeleitetem Graben wäre wohl im Interesse aller, auch da der Grundwasserspiegel im Gebiet der bereits ausgegrabenen Villa perspektivisch für die Erhaltung etwaiger Papyri ein unkalkulierbares Risiko ist ${ }^{109}$ und in Anbetracht der geographischen Lage der Ausgrabungen über die nächsten Jahrzehnte (kleinere) Erdbeben nicht ausgeschlossen sind (von Schlimmerem zu schweigen...), welche bereits ausgegrabene Strukturen der Villa schädigen und weitere Grabungen faktisch verhindern könnten. Zugegeben, hier spricht der erwartungsvolle Papyrologe und kein studierter Archäo-

109 Vgl. Guidobaldi und Esposito, „New Archaeological Research“ (wie Anm. 107), 21-22. 
loge, aber vielleicht könnten im Rahmen des archäologisch Vertretbaren zumindest versuchsweise kleinere Grabungen in der Umgebung von Räumen mit Buchrollenpotential vorgenommen werden. Dem „neuen Karl Weber“ (siehe 3.2) würde gewiss unsterblicher Ruhm winken! Da viele in Herkulaneum erprobte Verfahren und Innovationen für die gesamte Archäologie wegweisenden Charakter hatten und haben, ${ }^{110}$ sollte eine Lösung für den Spezialfall der Villa dei papiri und ihrer Bibliothek, welche die Suche nach weiteren Papyri einschließt, zu finden sein. Womöglich liefert ein etwaiger Erfolg des virtual unrolling (siehe 11) einen zusätzlichen Motivationsschub für neue Grabungen.

\subsection{Herculaneum Society}

Das Ziel weiterer Grabungen in der Villa mit (impliziter) Suche nach Papyri wird ebenfalls von der Herculaneum Society verfolgt. ${ }^{111}$ Dieser auch als Reaktion auf Gigantes Tod 2004 gegründeten, wachsenden Gesellschaft gehören interessierte Laien und renommierte Forscher an. Die Gesellschaft sitzt in Oxford, hat aber mittlerweile auch einen amerikanischen Zweig. Sie hält regelmäßig Kongresse in Herkulaneum oder anderen Orten ab und versucht sowohl die Geschichte Herkulaneums unter allen möglichen Aspekten zu studieren als auch die Forschungsergebnisse für eine breitere Öffentlichkeit $\mathrm{zu}$ kommunizieren. In der von der Gesellschaft initiierten Reihe Sozomena finden auch herkulanischpapyrologische Monographien und Sammelbände Platz. ${ }^{112}$ Neuigkeiten zu Herkulaneum erfährt man in ihrem regelmäßig erscheinenden Magazin (Newsletter of the Herculaneum Society).

110 Überblick über jüngste Entwicklungen bei Francesco Sirano, „Recenti studi e ricerche sull’ antica Ercolano: un aggiornamento nella prospettiva del nuovo Parco Archeologico," CErc 50 (2020): (311-336).

111 https://www.herculaneum.ox.ac.uk/. Ziele der Gesellschaft: „2. Research and Scholarship: the Society promotes research into all aspects of Herculaneum, including the continued investigation of the Villa of the Papyri and the publication of the results of research ...."

112 Etwa Zarmakoupi (Hg.), The Villa (wie Anm. 5) und Kilian Fleischer, The Original Verses of Apollodorus' Chronica (Berlin, 2020). 